\section{Subnanometer Translation of Microelectromechanical Systems Measured by Discrete Fourier Analysis of CCD Images}

\author{
Christophe Yamahata, Edin Sarajlic, Gijs J. M. Krijnen, and \\ Martin A. M. Gijs
}

\begin{abstract}
In-plane linear displacements of microelectromechanical systems are measured with subnanometer accuracy by observing the periodic micropatterns with a charge-coupled device camera attached to an optical microscope. The translation of the microstructure is retrieved from the video by phase-shift computation using discrete Fourier transform analysis. This approach is validated through measurements on silicon devices featuring steep-sided periodic microstructures. The results are consistent with the electrical readout of a bulk micromachined capacitive sensor, demonstrating the suitability of this technique for both calibration and sensing. Using a vibration isolation table, a standard deviation of $\sigma=0.13 \mathrm{~nm}$ could be achieved, enabling a measurement resolution of $0.5 \mathrm{~nm}(4 \sigma)$ and a subpixel resolution better than 1/100 pixel. [2010-0170]
\end{abstract}

Index Terms-Discrete Fourier transform (DFT), optical measurement, phase correlation, subpixel resolution.

Microelectromechanical systems (MEMS) comprise integrated devices made by batch-fabrication techniques, like those adopted from silicon-based integrated circuit technology. MEMS sensors and actuators have typical feature sizes ranging from a few tens of nanometers to several hundreds of micrometers. Mechanical transducers fabricated using silicon micromachining technology have to be characterized or even calibrated and possibly trimmed electronically before being used in an instrument. To mention only a few examples, the spring constant of an atomic force microscopy (AFM) cantilever, the voltage-displacement quadratic response of a comb-drive electrostatic actuator, or the sensitivity of a differential capacitive sensor are all parameters that are highly dependent on the microfabrication. Hence, at micro- and nanometer scales, precise and accurate dimensions can be difficult to guarantee because of process limitations. However, there are geometric parameters that are process independent, like the pitch of a periodic micropattern that is determined solely by the design. Thus, the periodicity can be used advantageously for precise and accurate position measurements.

Optical measurements are noninvasive in nature and constitute an alternative or a complement to solid-state sensors, which can be challenging to miniaturize and integrate. However, most optical techniques proposed for the characterization of static displacements, motion, and vibrations were developed to quantify the out-of-plane motion of MEMS structures, often relying on complex interferometric techniques [1]. In contrast, only very few approaches were demonstrated for high-resolution in-plane displacement measurements. With continuous illumination, the displacement of the moving part of a

Manuscript received June 10, 2010; accepted July 27, 2010. Date of publication September 9, 2010; date of current version October 1, 2010. This work was supported by the Swiss National Science Foundation through Ambizione under Grant PZ00P2_121827. Subject Editor M. Mehregany.

C. Yamahata and M. A. M. Gijs are with the Laboratory of Microsystems, École Polytechnique Fédérale de Lausanne, 1015 Lausanne, Switzerland (e-mail: christophe.yamahata@a3.epfl.ch).

E. Sarajlic is with SmartTip B.V., 7522 NB Enschede, The Netherlands.

G. J. M. Krijnen is with MESA+, University of Twente, 7500 AE Enschede, The Netherlands.

Color versions of one or more of the figures in this paper are available online at http://ieeexplore.ieee.org.

Digital Object Identifier 10.1109/JMEMS.2010.2067445
MEMS structure could be measured with an accuracy of 0.2 pixel, corresponding to a few tens of nanometers [2]. When stroboscopic illumination and advanced image processing techniques were used, a detection limit of a few nanometers could be reached [3], [4]. Lateral scanning confocal microscopy has been used to characterize the voltage-displacement response of a comb-drive actuator with a nanometer resolution [5]. Using a phase correlation algorithm, a displacement of $10-\mu \mathrm{m}$-scaled periodic micropatterned features with a high optical contrast could be detected with an accuracy of a few nanometers [6], [7].

We introduce here an extremely simple and robust method for measuring the in-plane linear displacement of MEMS microstructures with a subnanometer resolution. It is applicable to any transducer that shows a translational displacement and features periodic microstructures. Apart from the MEMS device, the setup consists of a charge-coupled device (CCD) camera attached to a bright field optical microscope and a computer for the analysis of the video sequences using MATLABbased dedicated software [8]. Optionally, a vibration isolation table can be used to reduce the mechanical noise and significantly enhance the resolution for subnanometer sensing. The working principle of our method is summarized in Fig. 1(a).

Periodic patterns must be realized to be a part of the moving structure (period $T_{\text {sens }}$ ). In order to improve the accuracy, it is advisable to include the periodic patterns on a reference structure (period $T_{\text {ref }}$ ). The only criterion for the choice of $T_{\text {sens }}$ and $T_{\text {ref }}$ is to preclude aliasing, which is guaranteed with spatial frequencies lower than the Nyquist frequency (half the sample frequency). Both structures are recorded simultaneously with the camera. Subsequently, the video frames are cropped to the pixel area of interest to analyze the absolute displacement of both structures separately. The displacement $\Delta x$ of the moving structure relative to the reference is simply obtained by subtraction.

The algorithm works as enumerated in the following sentences.

1) The pixel area of interest ( $M$ columns $\times N$ rows) is selected.

2) For each image frame $j$, a discrete intensity profile $f_{j}(m)$ is then calculated by averaging the gray value $g_{j}(m, n)$ of each pixel column: $f_{j}(m)=(1 / N) \sum_{n=0}^{N-1} g_{j}(m, n)$. The intensity profile of the first frame $f_{0}(m)$ serves as a reference for the calculations.

3 ) The displacement along the $x$-axis is calculated for each frame by phase correlation [3], [6], [7].

A subpixel resolution can be explained through the generalized discrete Fourier transform (DFT). From the shift theorem, we find the DFT of the shifted function $f_{0}(m-d)$ for $d \in \mathbb{Z}$

$$
F_{d}(k) \equiv \mathcal{F}\left\{f_{0}(m-d)\right\}=\mathcal{F}\left\{f_{0}(m)\right\} e^{-i \omega_{k} d}
$$

where $\omega_{k}=2 \pi k / M$ and $k=0,1, \ldots, M-1$. Equation (1) can be generalized to any real shift $d+\alpha$

$$
F_{d+\alpha}(k)=\mathcal{F}\left\{f_{0}(m)\right\} e^{-i \omega_{k}(d+\alpha)}
$$

where $\alpha \in \mathbb{R}:-1<\alpha<1$. Reciprocally, a discrete profile $f_{0}(m)$ can be shifted by $\Delta x$ by changing the phase $\varphi_{k}$ of its DFT, resulting in appropriate gray values at each of the sample points $f_{j}(m)$. We define $\Delta u$ as the width of a pixel (intersample distance along the $x$-axis) and write $\Delta x=\Delta u(d+\alpha)$. Assuming that $F_{d+\alpha}(k)=\mathcal{F}\left\{f_{j}(m)\right\}$, we deduce from (2)

$$
\varphi_{k}=\omega_{k}(d+\alpha)=\omega_{k} \frac{\Delta x}{\Delta u} .
$$


(a)

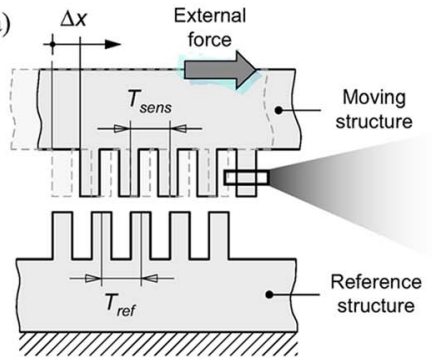

Pixels intensity

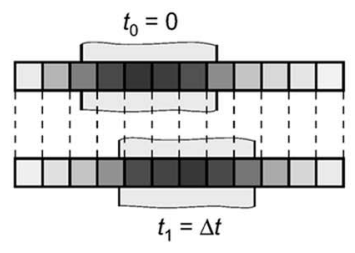

(b)

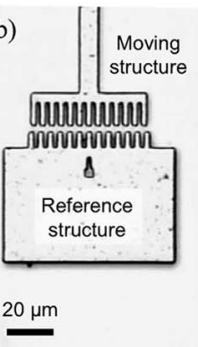

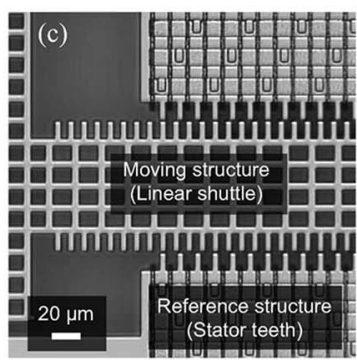

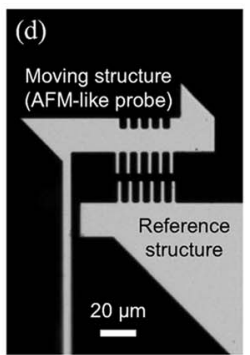

Fig. 1. (a) Principle of the measurement method. CCD camera attached to a microscope simultaneously records periodic patterns of a reference and of a moving structure (pitch $T_{\text {ref }}$ and $T_{\text {sens }}$, respectively). Motion-induced change in gray scale of pixels yields the displacement $\Delta x$, as calculated using DFT phase correlation. (b)-(d) Optical photographs of periodic structures etched in various MEMS devices. (b) Inchworm shuffle motor [9]. (c) Linear stepper motor [10]. (d) Microtweezers with an AFM-like probe [11].

TABLE I

CONFIGURATION OF THE EXPERIMENTAL SETUP

\begin{tabular}{cc}
\hline Equipment / Parameter & Model / Specifications \\
\hline Vibration isolation table & TMC 63-534 Lab Table \\
Digital microscope & Keyence VHX-600E \\
CCD image sensor & 1/1.8-inch, 2.11-million-pixel \\
Zoom lens & VH-Z500R $\left(\times 5000^{a}\right)$ \\
\hline & Windows AVI; progressive scan; \\
Video & $15 \mathrm{fps} ; 800 \mathrm{px} \times 480 \mathrm{px} ;$ \\
& $256(8$-bit $)$ levels of gray \\
Observation range & $61 \mu \mathrm{m} \times 36.6 \mu \mathrm{m}$ \\
Inter-sample pixel distance & $\Delta u \approx 75 \mathrm{~nm}$ \\
$T=T_{\text {ref }}=T_{\text {sens }}$ & $6 \mu \mathrm{m}$ \\
\hline${ }_{\text {Ratio between the apparent size on the 15-inch LCD monitor of the digital }}$ \\
microscope and the true size. \\
${ }^{b}$ Values obtained after calibration of the microscope by the manufacturer.
\end{tabular}

Equations (2) and (3) show that the displacement $\Delta x$ is retrieved with a subpixel resolution from the phase difference between $\mathcal{F}\left\{f_{j}(m)\right\}$ and $\mathcal{F}\left\{f_{0}(m)\right\}$.

In MATLAB, these calculations are performed straightforwardly with the functions "fft()" and "angle()" that calculate the DFT and the phase angle, respectively. In addition, since we only focus on the phase shift of the periodic patterns, we can increase the computation efficiency by limiting the DFT calculations to the corresponding spatial frequency index using the second-order Goertzel algorithm implemented with the function "goertzel()." Even though we are dealing with sequences of 2-D $(M \times N)$ images, we have been working only with 1 -D $(M \times 1)$ DFT functions. This is because the Fourier transform is linear. Thus, the phase calculated from $\mathcal{F}\left\{f_{j}(m)\right\}$ is identical to the average of the phases calculated for the $N$ Fourier transforms $G_{n}(k)=\mathcal{F}\left\{\left.g_{j}(m, n)\right|_{n}\right\}$ obtained for each individual line $n=0,1, \ldots, N-1$.

To validate our algorithm, we have tested it with various MEMS devices in which we have etched periodic microstructures. A few examples of such devices are shown in Fig. 1(b)-(d) and are described elsewhere in details [10], [11]. All the measurements presented hereinafter were performed on devices similar to the silicon microtweezers described in [12]. The main configuration parameters of the experimental setup used to collect these data are summarized in Table I.

In Fig. 2, we show the typical results obtained with an electrostatic comb drive that was actuated sequentially with very low voltages to achieve nanometer-scale displacements. We clearly see the benefit of using a reference probe to decrease the standard deviation $\sigma$ by a factor greater than three and to reach a subnanometer resolution of $4 \sigma \approx 0.5 \mathrm{~nm}$. Bearing in mind that the fundamental limitations on the image registration and resolution are different, it is noteworthy that the achieved displacement resolution is far beyond the diffraction limit of optical imaging systems (the latter being on the order of the wavelength of visible light, typically $400-700 \mathrm{~nm}$ ) [3]. With $\Delta u \approx 75 \mathrm{~nm}$,

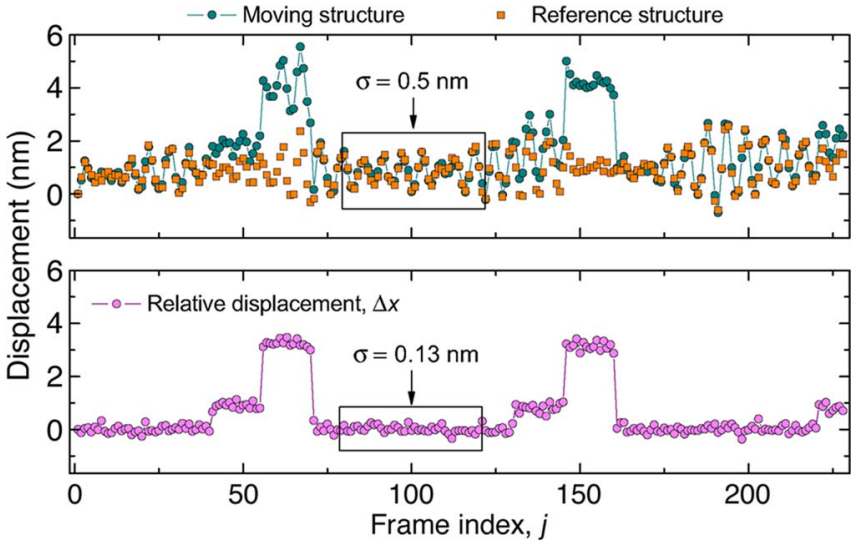

Fig. 2. Demonstration of subnanometer displacement resolution of a comb drive. (Top) Absolute displacement of the moving and reference structures. (Bottom) Relative displacement $\Delta x$ obtained by subtracting the reference data from the absolute displacement data.

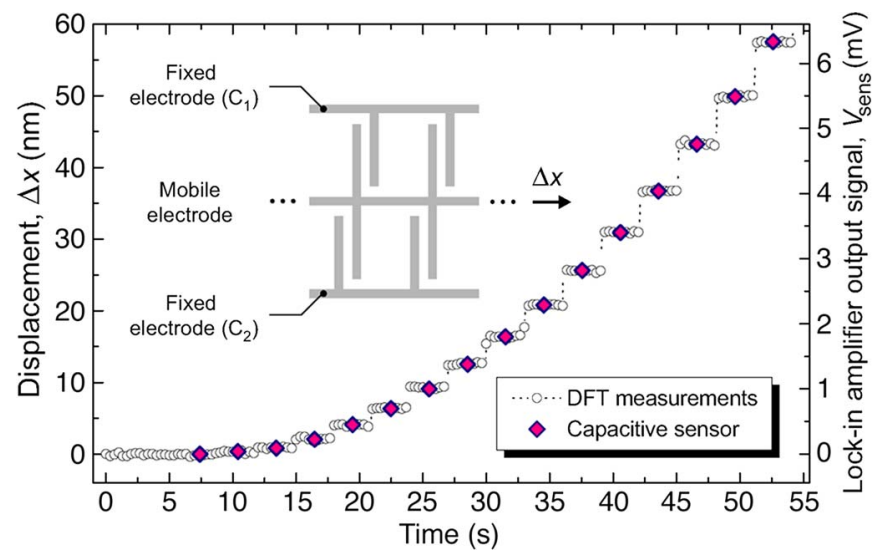

Fig. 3. Output signal of a capacitive sensor $V_{\text {sens }}$ versus time upon actuation with a comb drive and comparison with the displacement $\Delta x$ measured by DFT method. Insert is a schematic of the capacitive sensor.

this performance corresponds to a subpixel resolution better than 1/100 pixel. In Fig. 3, we have recorded the output signal of a differential capacitive sensor monitored using a lock-in amplifier system [12] and compared these results with our DFT phase correlation method. The displacement was induced by a comb drive using a sequence of incremental constant voltage steps. The output signal of the sensor $V_{\text {sens }} \propto \Delta C \equiv C_{1}-C_{2} \propto \Delta x$ [12] is dependent on the dimensions of the etched microstructures (gap width) while the measurements obtained with the DFT method are insensitive to the process variations. 
Number of columns, $M$ 80160240320400480560640720800
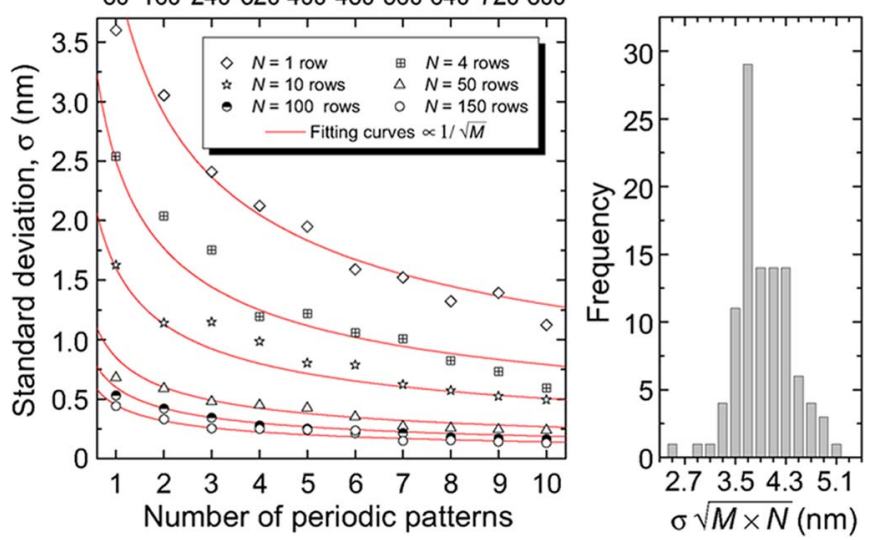

Fig. 4. (Left) Standard deviation $\sigma$ as a function of the size of the video frame ( $M \times N$ pixels). (Right) Histogram of $\sigma \sqrt{M \times N}$ using the data from the left panel.

Hence, this result shows a direct application of our method for the straightforward calibration of the capacitive sensor.

In addition to these measurements, we have verified the robustness of the algorithm. We found that the systematic error due to the angular misalignment $\left( \pm 2^{\circ}\right)$ was smaller than $1 \%$. Finally, we measured the influence of the number of pixels $(M \times N)$ on the standard deviation $\sigma$. Fig. 4 shows that $\sigma$ decreases both with $\sqrt{M}$ and $\sqrt{N}$, as what is expected for the standard deviation of a distribution of uncorrelated random samples. The histogram of Fig. 4 reveals an average standard deviation per pixel of $\approx 4 \mathrm{~nm}$.

To summarize, the main reasons for the unprecedented subnanometer resolution are as follows: 1) the exploitation of multiple periodic microstructures in combination with a Fourier phase-shift algorithm; 2) the presence of an in situ measurement reference to eliminate the errors induced by parasitic motion; 3 ) the use of a high-end optical microscope and a CCD camera with a large number of pixels; 4) the use of an antivibration table to reduce the mechanical noise; and 5) a homogeneous illumination and a good optical contrast between the periodic patterns and the background. The proposed method is extremely robust and can be implemented in a straightforward way for any micromechanical device. It is to be mentioned that its use is extensible to in-plane Cartesian displacements (simultaneous displacements along the $x$ and $y$-axes, without rotation). Accurate measurements are not dependent on the MEMS micromachining but instead on the well-defined periodicity of the repeating patterns. Last but not least, the accuracy is not compromised by the observation variables, for example, the use of an ill-calibrated microscope, because the welldefined period of the repeating patterns in the mask design serves as the absolute reference unit for the DFT algorithm.

\section{REFERENCES}

[1] A. Bosseboeuf and S. Petitgrand, "Characterization of the static and dynamic behaviour of M(O)EMs by optical techniques: Status and trends," J. Micromech. Microeng., vol. 13, no. 4, pp. S23-S33, Jun. 2003.

[2] D. J. Burns and H. F. Helbig, "System for automatic electrical and optical characterization of microelectromechanical devices," J. Microelectromech. Syst., vol. 8, no. 4, pp. 473-482, Dec. 1999.

[3] C. Q. Davis and D. M. Freeman, "Using a light microscope to measure motions with nanometer accuracy," Opt. Eng., vol. 37, no. 4, pp. 12991304, Apr. 1998.

[4] P. Sandoz, J. M. Friedt, and E. Carry, "In-plane rigid-body vibration mode characterization with nanometer resolution by stroboscopic imaging of a microstructured pattern," Rev. Sci. Instrum., vol. 78, no. 2, p. 023706 , Feb. 2007.

[5] Z. Li, K. Herrmann, and F. Pohlenz, "Lateral scanning confocal microscopy for the determination of in-plane displacements of microelectromechanical systems devices," Opt. Lett., vol. 32, no. 12, pp. 1743-1745, Jun. 2007.

[6] P. Sandoz, J. C. Ravassard, S. Dembélé, and A. Janex, "Phase-sensitive vision technique for high accuracy position measurement of moving targets," IEEE Trans. Instrum. Meas., vol. 49, no. 4, pp. 867-872, Aug. 2000

[7] P. Sandoz, V. Bonnans, and T. Gharbi, "High-accuracy position and orientation measurement of extended two-dimensional surfaces by a phasesensitive vision method," Appl. Opt., vol. 41, no. 26, pp. 5503-5511, Sep. 2002.

[8] The MATLAB GUI can be downloaded free of charge at. [Online]. Available: http://lmis2.epfl.ch/nanoplus/

[9] E. Sarajlic, E. Berenschot, N. Tas, H. Fujita, G. Krijnen, and M. Elwenspoek, "High performance bidirectional electrostatic inchworm motor fabricated by trench isolation technology," in Proc. Transducers, Seoul, South Korea, 2005, pp. 53-56.

[10] E. Sarajlic, C. Yamahata, M. Cordero, and H. Fujita, "An electrostatic 3-phase linear stepper motor fabricated by vertical trench isolation technology," J. Micromech. Microeng., vol. 19, no. 7, p. 074 001, Jun. 2009.

[11] C. Yamahata, E. Sarajlic, L. Jalabert, M. Kumemura, D. Collard, and H. Fujita, "Mechanical characterization of biomolecules in liquid using silicon tweezers with subnanonewton resolution," in Proc. IEEE MEMS, Sorrento, Italy, 2009, pp. 607-610.

[12] C. Yamahata, D. Collard, B. Legrand, T. Takekawa, M. Kumemura, G. Hashiguchi, and H. Fujita, "Silicon nanotweezers with subnanometer resolution for the micromanipulation of biomolecules," J. Microelectromech. Syst., vol. 17, no. 3, pp. 623-631, Jun. 2008. 